\title{
Direct Observation of Trapping Motion in Elongated Fourier-Transform Mass Spectrometry Trapped Ion Cells
}

\author{
Steven A. Hofstadler and David A. Laude, Jr. \\ Department of Chemistry, The University of Texas at Austin, Austin, Texas, USA
}

\begin{abstract}
Measurements by Fourier-transform mass spectrometry (FTMS) have been used to measure trapping oscillation profiles in elongated trapped ion cells of length $10-43 \mathrm{~cm}$. Trapping periods extracted from these profiles are found to vary linearly with cell length for elongated cells. This is in contrast with the prediction based on a quadrupolar approximation of the electric field that trapping period should increase exponentially with increased cell length. An alternate analytical expression for trapping motion is derived that better accounts for the motion of ions with sufficient energy to approach the trap plates. Calculated trapping frequencies are within a few percent of values determined from ion trajectory simulations for any combination of cell length, trap potential, ion mass, and ion kinetic energy. The new expression also explains the experimentally determined trapping data obtained in elongated cells. This expression predicts an average axial energy near $0.6 \mathrm{eV}$ for the ions that are preferentially detected by FTMS with the specific pulse sequence employed. (I Am Soc Mass Spectrom 1990, 1, 351-360)
\end{abstract}

$\mathrm{I}$ Fourier transform ion cyclotron resonance mass spectrometry (FTMS), it is necessary to establish an electric field between parallel trap plates positioned perpendicular to the magnetic field to constrain the $z$ axis molion of ions in the trapped ion cell. This trapping electric field and the resulting trapping motions are continually scrutinized in an effort to improve the analytical performance of FTMS. For example, mass calibration equations now correct for the reduction in measured cyclotron frequency imposed by radially inhomogeneous trapping fields [1-3]. Marshall and coworkers $[4,5]$ have shown that the radially inhomogeneous trapping field imposes an upper limit on the mass of an ion that can be confined in the cell. Approaches taken to minimize the deleterious effects of the radial trapping field include improving cell geometries, as with the hyperbolic cell design $[6,7]$, increasing the cell dimension along the $z$ axis [8], inserting grounded screen inserts adjacent to the trap plates [5]. adiabatically increasing trapping potentials prior to ion excitation [9], and developing shimmed ion cells [10, 11].

As described above, an understanding of the $z$-axis motion of ions in the trapping electric field is of importance to optimal FTMS performance, yet with few exceptions [4, 10-12] a quadrupolar approximation of these fields has been employed in developing models to describe the ion motion. If it is assumed that ions

Address reprint requests to David A. Laude, Jr., Department of Chemistry, The University of Texas at Austin, Austin, TX 78712. are constrained near the bottom of the potential well, then the approximation yields an effective model of the trapping motion that simplifies the arduous task of modeling radial ion motion. Of particular relevance to the present work is the derivation of an equation for trapping motion in elongated cells presented by Hunter et al. [8] that is based upon the quadrupolar approximation and makes the assumption ions are constrained to the center of the cell. However, for cells of increasing length and for ions with increasing amplitude in the well, the equation becomes ineffective.

Better models of the trapping motion in cells of any dimension have not been developed because it is numerically cumbersome to adequately evaluate potentials in longer cells. Additionally, few direct measurements of trapping frequency are presented in the literature, and many support models derived from quadrupolar approximations of the electric fields. For example, trapping frequency data acquired under conditions that approximate harmonic motion include the study of Beauchamp and Armstrong [13] of the z-axis ejection of $\mathrm{Ar}^{+}$from an ion cyclotron resonance cell and the demonstration of Schweikhard et al. [14] of a multiplex detection scheme for the trapping motion in a Penning ion trap. However, experiments by Giancaspro and co-workers $[15,16]$ and Hunter et al. [8] have yielded data that would not necessarily be expected to exhibit harmonic motion. In the work of Giancaspro and co-workers, trapping oscillations between adjacent trapped ion cells were observed during FTMS studies of mass discrimination during ion transfer, but were only indicated to approximate pre- 
dicted values. This dual cell oscillation has been modeled [17] by using a potential expression along the $z$ axis and a fourth order Runge-Kutta solution of the equation of motion in order to investigate the anharmoric nature of the dual cell when the conductance limit is grounded. In studies of ion motion in elongated trapped ion cells, Hunter et al. correlated shifts in cyclotron frequency with trapping voltage, but were probably detecting ions confined to the center region of the cell where the quadrupolar model is most effective. Recently, Van De Guchte and Van Der Hart [12] applied a fourth order Runge-Kutta solution to investigate the trapping motion in elongated trapped ion cells and showed that the trapping frequency in elongated cells is dependent on both the $z$ amplitude of the oscillation and the position of the ion in the $x y$ plane.

It is likely that the inadequacies of the quadrupolar approximation will become increasingly apparent with increased use of elongated trapped ion cells [18] and ion sources and injection techniques that introduce high kinetic energy ions to the trapped ion cell for FTMS detection [18-20]. For example, recently we examined the combination of suspended trapping pulse sequences with variable length external ion reservoirs to alleviate space charge distortion in FTMS spectra and to examine the spatial and kinetic energy distribution of ions detected by FTMS [20-22]. In the course of this work, trapping frequencies of ions in elongated cells were measured directly by the method of Giancaspro and co-workers $[15,16]$ and found to be in conflict with predicted values from the model of Hunter et al. [8] for trapping motion in such cells. Here, in an effort to reconcile this disagreement, an alternate model for trapping motion in trapped ion cells of any length is developed. This new model is based not on the quadrupolar approximation of the electric field, but rather on an analytical solution of the Lorentz equation that uses an improved estimate of the electric field in rectangular trapped ion cells. The resulting expression for trapping motion explains the discrepancies between the quadrupolar model and our data, and generates trapping frequency values for any given ion kinetic energy, mass, cell length, and trapping potential. The

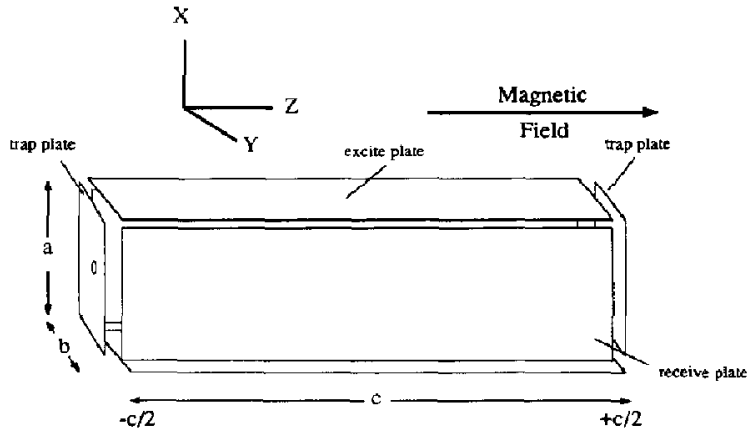

Figure 1. Coordinate axis and cell orientation of orthorhombic trapped ion cell. The applied magnetic field is along the $z$ axis and trapping potentials are applied to the trap plates located at $\pm c / 2$.

calculated frequencies are within a few percent of values obtained with ion trajectory simulations that use sophisticated numerical methods to estimate exact values for the electric field.

\section{Theory}

The model for trapping oscillations is developed for an orthorhombic trapped inn cell with sides of dimension $a, b$, and $c$ oriented in a Cartesian coordinate system with coordinate origin in the center of the cell, as shown in Figure 1. Trapping motion is along the $z$ axis of a trapped ion cell centered in the $z=0$ plane between trap plates positioned at $\pm c / 2$. A uniform magnetic field, $B$, applied along the $z$ direction constrains radial motion of the ions in cyclotron orbits. Trapped ion cell potentials are $V_{0}$, which is applied to the two sets of parallel plates positioned colinear with $B$ that serve as transmit and receive plates for the trapped ion cell, and $V_{T}$, which is applied to two trap plates positioned perpendicular to $B$.

Following established procedures for solving the equations of motion of ions detected by FTMS in an orthorhombic cell, the exact potential at any point in the cell is expressed in a form derived by Sharp et al. [23]

$$
V_{x, y, z}=V_{0}+16 \pi^{-2}\left(V_{T}-V_{0}\right) \sum_{m=0}^{\infty} \sum_{n=0}^{\infty} \frac{\cosh (k \pi z / c)(-1)^{m+n} \cos \left[(2 m+1) \frac{\pi x}{a}\right] \cos \left[(2 n+1) \frac{\pi y}{b}\right]}{\cosh (k \pi / 2)(2 m+1)(2 n+1)}
$$

with

$k=\left[\left\{(2 m+1)\left(\frac{c}{a}\right)\right\}^{2}+\left\{(2 n+1)\left(\frac{c}{b}\right)\right\}^{2}\right]^{1 / 2}$

Because for this work we are interested in derivation of an expression for motion along the $z$ axis, the general equation can be simplified by setting $x=y=0$. Equation 1 is reduced further because $a=b$ in usual
FTMS cell configurations, and with $V_{0}=0.0$, becomes

$$
V_{z}=16 \pi^{-2} V_{T} \sum_{m=0}^{\infty} \sum_{n=0}^{\infty} Q \cosh \left(k^{\prime} \pi z / a\right)
$$

with

$$
k^{\prime}=\left\{[(2 m+1)]^{2}+[(2 n+1)]^{2}\right\}^{1 / 2}
$$


and

$$
Q=\frac{(-1)^{m+n}}{(2 m+1)(2 n+1) \cosh \left(k^{\prime} \pi c / 2 a\right)}
$$

Although considerably simpler than eq 1 , eq 3 still yields exact potentials for the important case of ions formed along the center line of a cell between $-\frac{c}{2} \leq z \leq \frac{c}{2}$, as, for example, during electron ionization. As will be demonstrated, eq 3 provides access to an improved analytical solution for the trapping motion along the center line of elongated trapped ion cells.

Harmonic oscillator solution. Before deriving a new expression for the trapping motion, it is instructive to obtain an equation for trapping motion through the quadrupolar approximation. Equation 3 is first rewritten as a Taylor expansion of the hyperbolic cosine term in the numerator. Although a close approximation of the potential is obtained by adding sufficient terms to the Taylor series as the cell is lengthened, the assumption is usually made that the potential, at least in the center of the cell, is effectively described by the first two terms of the expansion, which yields eq 6 upon rearrangement.

$$
\begin{aligned}
V_{z}= & 16 \pi^{-2} V_{T} \sum_{m=0}^{\infty} \sum_{n=0}^{\infty} Q \\
& +16 \pi^{-2} V_{T} z^{2} \sum_{m=0}^{\infty} \sum_{n=0}^{\infty} \frac{Q k^{12} \pi^{2}}{2 a^{2}}
\end{aligned}
$$

Importantly, $z^{2}$ is brought outside the double summation. An expression for the electric field is now obtained from the negative derivative

$$
E=\frac{-d V}{d z}=-32 \pi^{-2} V_{T} z \sum_{m=0}^{\infty} \sum_{n=0}^{\infty} \frac{Q k^{2} \pi^{2}}{2 a^{2}}
$$

and the Lorentz equation for an ion traveling in a uniform magnetic field is then

$$
\frac{d^{2} z}{d t^{2}}=-\omega_{T^{2}} z
$$

with a trapping frequency, $\omega_{T}$, given by

$$
\omega_{T}=\left(\frac{q 16 V_{T} \sum \sum Q k^{n} \pi^{2}}{m a^{3}}\right)^{1 / 2}
$$

with $q$ the charge of the ion, and $m$ the mass of the ion. This expression is analogous to that derived by Hunter et al. [8] to describe the trapping frequency, $\omega_{T}$, in an elongated well. Specifically, the cell geometry constant $\beta$ in that work is equivalent to $\sum \sum 8 Q k^{2} \pi^{2}$

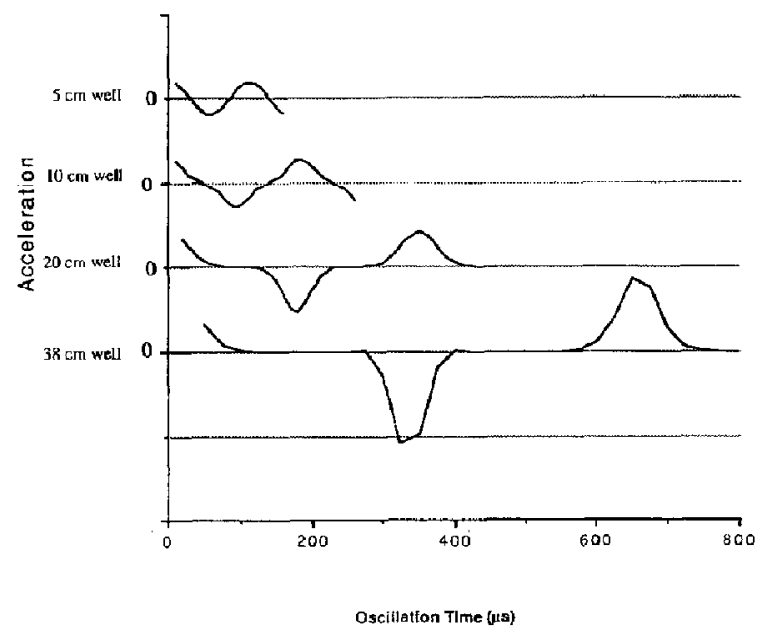

Figure 2. Ion trajectory calculations of acceleration for a $1.0-\mathrm{eV}$ ion in a $2.0-\mathrm{V}$ well as a function of time. Trajectories are determined from SIMION calculations for cells of length 5 to $38 \mathrm{~cm}$.

here. Equation 8 is easily solved for velocity, $v_{z}$, with a constant of integration, $z_{0}$, corresponding to the initial position of an ion in the cell between $\pm c / 2$.

$$
v_{z}= \pm \omega_{T}\left(z_{0}^{2}-z^{2}\right)^{1 / 2}
$$

Note especially that eq 9 indicates trapping frequency falls off markedly with increasing cell length, $c$, because $Q$ decreases as $(\cosh (c / a))^{-1}$. For example, in a cubic cell with $a=b=c=5 \mathrm{~cm}$, a cell geometry factor $\beta$ of 2.77 yields $\omega_{T}=57.8 \times 10^{3} \mathrm{~Hz}$ at $V_{T}=2.0$ $V$ for an ion of $\mathrm{m} / \mathrm{z} \mathrm{128}$; for a $5 \times 5 \times 25 \mathrm{~cm}^{3}$ cell, $\beta$ reduces to $4.8 \times 10^{-4}$, and a trapping frequency of 761 $\mathrm{Hz}$ is predicted.

Cosh function approximation. In contrast with predictions based upon the harmonic oscillator model, it can be argued that any model for trapping motion in elongated wells should demonstrate that as the length of the well increases, ions behave with increasing particle-in-a-box character. Trapping period is then strongly dependent on the initial kinetic energy of the ion. Such motion is easily distinguished from harmonic motion because ions in the center of the well would achieve a constant velocity and zero acceleration. To demonstrate this characteristic of trapping motion in elongated wells, an ion trajectory program [24] was used to simulate the $z$-axis flight of an ion with $1.0-\mathrm{eV}$ initial kinetic energy traveling along the $z$ axis in the center of a cell with $2-\mathrm{V}$ trap potentials. The acceleration is plotted as a function of oscillation time for cells ranging in size from $5 \mathrm{~cm}^{3}$ to $5 \times 5 \times 38 \mathrm{~cm}^{3}$, as shown in Figure 2. As suspected, the ion spends a larger fraction of an oscillation period at constant velocity as the aspect ratio increases. 
The purpose of this work is to produce an alternate model for longitudinal ion motion along the center of the cell that provides a rapid assessment of trapping period in elongated cells for ions with any total energy. As a starting point for derivation of a new expression, rather than substitute a Taylor expansion of the cosh term, an exact solution for the electric field from the given potential in eq 3 is obtained.

$$
E=\frac{d V}{d z}=-16 \pi^{2} V_{\mathrm{T}} \sum_{m=0}^{\infty} \sum_{n=0}^{\infty} \frac{k^{\prime} \pi Q}{a} \sinh \left(k^{\prime} \pi z / a\right)
$$

From inspection of eq 1 , at the boundary $V_{2}$ must equal $V_{T}$ and thus

$$
\lim _{m, n \rightarrow \infty} \sum_{m=0}^{\infty} \sum_{n=0}^{\infty} \frac{(-1)^{m+n}}{(2 m+1)(2 n+1)}=\frac{\pi^{2}}{16}
$$

This convergence constant is now substituted into eq 11 resulting in an approximation of the electric field that is accurate at the boundary where $V_{z}=V_{T}$. Equation 11 is now reduced to

$$
E=-V_{T} \sum_{m=0}^{\infty} \sum_{n=0}^{\infty} \frac{k^{\prime} \pi \sinh \left(k^{\prime} \pi z / a\right)}{a \cosh \left(k^{\prime} \pi c / 2 a\right)}
$$

Unfortunately, $z$ is not easily extracted from the double summation as was possible following substitution of Taylor expanded terms. Instead, the electric field is further approximated as the first term of the double summation, $m=n=0$. The constant, $k^{\prime}$, in eq 4 , now equals $\sqrt{2}$, and eq 13 simplifies further to

$$
E=-\frac{\sqrt{2} \pi V_{T} \sinh (\sqrt{2} \pi z / a)}{a \cosh (\pi c / \sqrt{2} a)}
$$

It should be pointed out that because of the truncated infinite summation and convergence constant substitution, eq 14 is not an exact expression. The limitations of these simplifications are more pronounced for cells with small aspect ratios but become insignificant as the aspect ratio of the cell increases. This limitation for smaller aspect ratios is not particularly problematic because in such cases the quadrupolar approximation provides an acceptable solution.

As an alternative to using the convergence constant at the boundary, in which $k^{\prime}=\sqrt{2}$, a variable exponential term can be employed to obtain a more exact solution, For example, a fitting procedure could be implemented to obtain an expression analogous to eq 14

$$
E=-\frac{\xi V_{T} \sinh (\xi z / a)}{a \cosh (\xi c / 2 a)}
$$

in which $\xi$ is an arbitrary parameter fit to the exact electric field. This is shown in eq 15. This approach is more cumbersome, however, as it requires an additional numerical fitting step before it can be implemented. The advantage of eq 14 is that, despite its simplicity, a surprisingly accurate equation of motion for elongated cells can be generated, as shown below.

The solution for an equation of motion of the form

$$
\frac{d^{2} z}{d t^{2}}=-A \sinh (B z)
$$

in terms of velocity is given by

$$
v_{z}= \pm\left\{2 \frac{A}{B}\left(\cosh \left(B z_{0}\right)-\cosh (B z)\right\}^{1 / 2}\right.
$$

Simplifying terms, the trapping motion velocity expression is then

$$
\begin{aligned}
v_{z}= & \pm\left\{\left(\frac{2 q V_{t}}{m \cosh (\pi c / \sqrt{2} a)}\right)\right. \\
& \left.\times\left(\cosh \left(\frac{\sqrt{2} \pi z_{0}}{a}\right)-\cosh \left(\frac{\sqrt{2} \pi z}{a}\right)\right)\right\}^{1 / 2}
\end{aligned}
$$

The constant of integration, $z_{0}$, defines the starting position and determines the total energy of the ion in the well.

Numerical integration of eq 18 , with limits of integration $z=z_{0}$ to $z=0$, yields one quarter of the oscillation period for an ion of a specific total energy. This expression was reached by truncating an infinite summation, and in that respect is similar to the quadrupolar approximation. As will be demonstrated, however, the $(\cosh z)^{1 / 2}$ function of eq 18 is an effective, yet still manageable, alternative to the quadrupolar solution in eq 9 for estimating the trapping frequency of axial ions in elongated FTMS trapped ion cells. We point out that previously Chesnavich et al. [25] employed an ion velocity expression for trapping motion in ion cyclotron resonance (ICR) cells that is similar to eq 18 ; however, in that case arbitrary constants defined in the function were a best fit of experimentally generated electric field data.

\section{Experimental}

The FTMS vacuum chamber and electrode configuration with which oscillations in elongated trapping wells were measured are shown in Figure 3. Experiments were performed in a $10-\mathrm{cm}$ diameter $\times 80-\mathrm{cm}$ length stainless steel vacuum chamber housed in the bore of a 3.0 T solenoidal-shaped superconducting magnet. The vacuum chamber was pumped to a base pressure of $2 \times 10^{-9}$ torr with two $700 \mathrm{~L} / \mathrm{s}$ diffusion pumps. An electron gun positioned $48 \mathrm{~cm}$ from the center point 

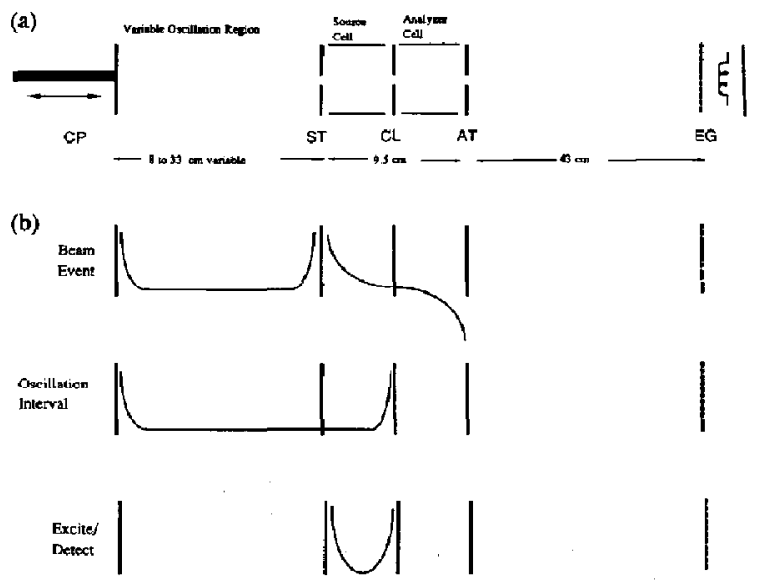

Figure 3. (a) Electrode configuration in the FTMS vacuum system. The ion reservoir was variable in length to $33 \mathrm{~cm}$. (b) FTMS pulse sequence used to determine trapping frequency with the jon cloud formed in the region between $\mathrm{CP}$ and ST, before oscillating in the larger reservoir between CP and CL, with FTMS detection in the source side trapped ion cell defined by ST and CL.

of the magnet bore was used for ionization. Electron currents between $200 \mathrm{nA}$ and $20 \mu \mathrm{A}$ were focused by the magnetic field to less than a $2-\mathrm{mm}$ diameter at the strongest point in the magnetic field and traversed the length of the vacuum chamber. A series of five stainless steel plates or grids were positioned perpendicular to the magnetic field to direct longitudinal ion and electron motion. Electrode EG gates. the electron beam into the magnet bore during a beam event. Electrodes AT, CL, and ST comprise a dual cell assembly centered in the homogeneous region of the magnet. Holes $2-\mathrm{mm}$ to $5-\mathrm{mm}$ in diameter in the center of the plates allow free passage of charged particles with sufficiently small cyclotron radius along the length of the vacuum chamber. The 5-cm region between AT, the analyzer trap plate, and CL, the conductance limit, is configured with two sets of plates parallel to the magnetic field to form a cubic trapped ion cell in which FTMS detection is performed. Similarly, the region between ST, the source trap, and CL is configured to permit FTMS detection in an adjacent higher pressure region of the chamber. The $2-\mathrm{mm}$ diameter orifice at electrode CL permits a sample-dependent pressure differential of $10^{2}$ and $10^{3}$ to be achieved. A final electrode, CP, serves a dual function as collector plate for the electron beam and as a trap plate for an external reservoir. Electrode CP is both probe mounted and electrically isolated to allow formation of external reservoirs between CP and ST with lengths variable between $5 \mathrm{~cm}$ and $38 \mathrm{~cm}$. Potentials applied to electrodes $\mathrm{CP}, \mathrm{ST}$, $C L$, and AT during each FTMS pulse sequence are independently variable between $-9.75 \mathrm{~V}$ and $+9.75 \mathrm{~V}$.

Trapping oscillations in variable length reservoirs are observed directly with an FTMS pulse sequence and electrode configuration, as shown in Figure $3 b$. This pulse sequence was first applied by Giancaspro and co-workers $[15,16]$ for selectively transferring ions of different mass between two trapped ion cells. Here, for most experiments, electrodes CP and CL define the oscillation region length, $c$, and $\mathrm{ST}$ serves as an interior electrode with which to create an initial ion population in a smaller trapping well. During the beam, electrode potentials are adjusted to retain ions exclusively in the potential well between electrodes $\mathrm{CP}$ and ST. During the subsequent suspended trapping event, electrode ST is set to $0.0 \mathrm{~V}$ to form the larger well and begin oscillation of the ion cloud. At increasing time intervals following suspension of the trapping voltage, the electrodes' potentials are again altered to trap the ion population present between ST and CL for FTMS detection. Data acquisition at suspended trapping intervals of 20 to $50 \mu \mathrm{s}$ was sufficient to adequately define the trapping oscillations in a profile acquired over a several millisecond time period. Experiments were commonly performed with $2-\mathrm{V}$ potentials applied to trapping electrodes during the beam event, the oscillation period, and detection.

The molecular ion of naphthalene at mass 128 was used for most experiments, the exception being experiments with PFT'BA performed to evaluate mass dependence of the trapping oscillation. Naphthalene was selected for its nonreactivity and because the molecular ion is the dominant ion in the electron ionization spectrum. The sample was introduced through a variable leak valve to pressures of $1 \times 10^{-8}$ torr above background in the source vacuum chamber. A $20-\mathrm{eV}$ electron ionization voltage, 5-ms beam event time, and $2-\mu \mathrm{A}$ beam current through the cell generated initial ion populations that exhibited minimal effects from ion coulombic repulsion. Broadband FTMS detection over an $800 \mathrm{kHz}$ bandwidth with $16 \mathrm{~K}$ data points followed broadband excitation over a $2.66 \mathrm{MHz}$ bandwidth at a $3200 \mathrm{~Hz} / \mu \mathrm{s}$ sweeprate. Each stored file of ten coadded scans was processed by baseline correction, sinebell apodization, and magnitude mode Fourier transformation.

\section{Results and Discussion}

Irapping sequence validation. To assess the validity of the pulse sequence and electrode configuration shown in Figure 3 for determining trapping frequency, several control experiments and ion trajectory simulations were performed. An important consideration to be addressed is whether the grounded interior electrode significantly perturbed the trapping oscillation. In order to evaluate this effect the ion trajectory program was used to calculate exact potentials for a $5 \times 5 \times 10 \mathrm{~cm}^{3}$ trapped ion cell along the center line of the cell both with and without a grounded center electrode. With 2-V potentials applied to the exterior trap plates, the grounded interior electrode altered the potential in the 

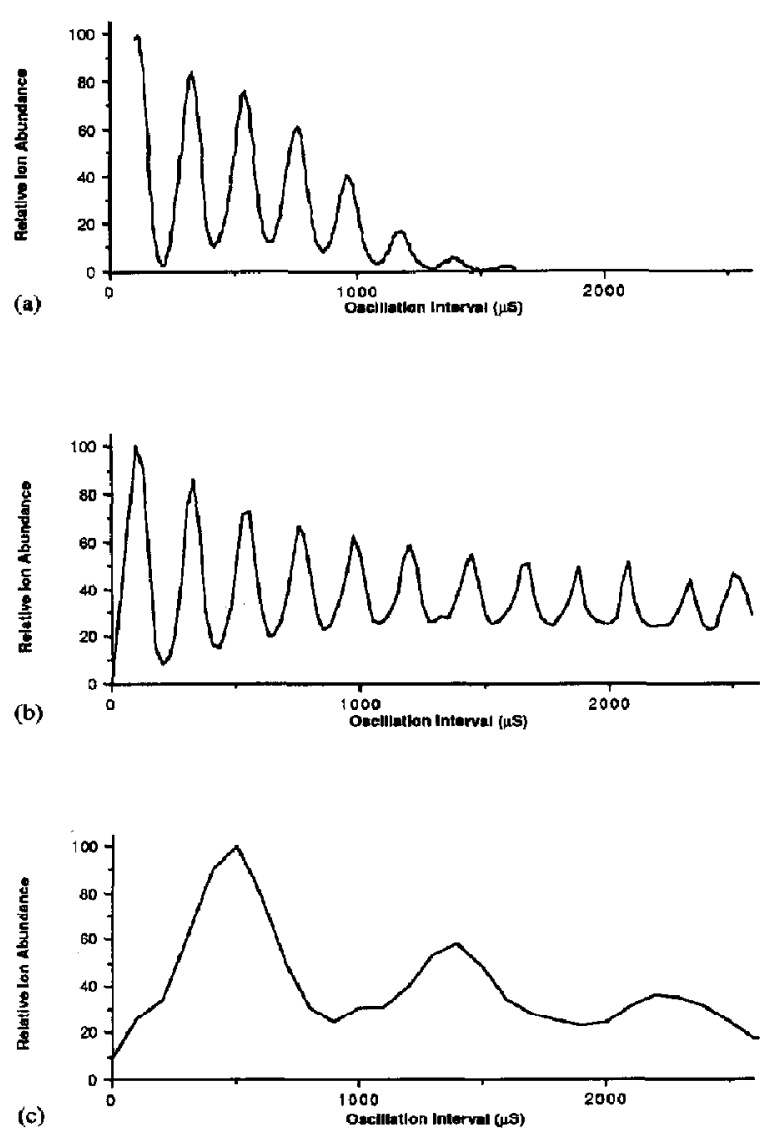

Figure 4. Ion profiles exhibiting oscillations corresponding to trapping period. (a) Oscillations in the $10-\mathrm{cm}$ region between electrodes ST and AT of the dual cell with detection in the analyzer cell. (b) Oscillations in a $10-\mathrm{cm}$ region between $\mathrm{CP}$ and CL with detection in the source cell. (c) Oscillations in a 43-cm region between $\mathrm{CP}$ and $\mathrm{AT}$ with detection in the analyzer trap.

center of the $10-\mathrm{cm}$ cell by less than $70 \mathrm{mV}$. Ion trajectory simulations of the trapping period for an $m / z$ 128 ion with $0.5-\mathrm{eV}$ initial kinetic energy were then determined to be $208 \mu \mathrm{s}$ with, and $206 \mu \mathrm{s}$ without the grounded electrode. The trapping frequency for an $\mathrm{m} / \mathrm{z}$ 128 ion in the $2-\mathrm{V}$ well of the $10-\mathrm{cm}$ dual cell was next determined experimentally by mimicking Giancaspro and co-workers' [15] uriginal procedure; the resulting profile in Figure 4a exhibits a trapping oscillation period of $218 \mu$ s between electrodes ST and AT, which is in good agreement with the ion trajectory simulation.

Another important consideration that must be addressed is whether the external reservoir was effectively approximated by the potential expression in eq 1 , despite the absence of excite and detect plates. Measurement of trapping oscillations in elongated cells would be simplified by using the variable length ion reservoir formed between electrodes CP and ST. Unfortunately, the absence of parallel excite and detect plates that traverse the reservoir length is in conflict with the orthorhombic cell geometry in eq 1, which is the foundation for trapping motion derivations. The actual geometry in the $x$ and $y$ dimensions of the external reservoir is instead approximated by the $10-\mathrm{cm}$ diameter cylinder that serves as the vacuum chamber housing. It would be expedient if, for the specific cylindrical and orthorhombic cell geometries in question, ions traveling along the center line at $x=y=0$ would experience approximately equal electric fields. This would validate a comparison of external reservoir experimental data with the derived equations. Given the relative similarity of cell sizes and symmetries, a similarity of electric fields would not be a surprising result, and simulated potential maps for the two geometries in fact differ by less than $100 \mathrm{mV}$ along the midpoint of the center line. Ion trajectory studies of the trapping period for both geometries are also in good agreement. For experimental verification, the trapping oscillation profile for a $10-\mathrm{cm}$ well formed between electrodes CP and CL was obtained and, as shown in Figure $4 b$, closely approximates the $10-\mathrm{cm}$ elongated trapped ion cell with grounded excite and detect plates shown in Figure 4a. Similar experiments using the 69, 131, and 219 mass ions of PFTBA with several trapping potentials were performed and the observed trapping oscillation profiles between $\mathrm{CP}$ and $\mathrm{CL}$, and ST and AT were in excellent agreement. We assume with some confidence then, that application of the suspended trapping pulse sequence in Figure 3 will yield trapping oscillation data that approximate the actual trapping motion of ions in elongated trapped ion cells. It should be noted, however, that this assumption pertains only to this particular experimental configuration; a vacuum chamber of significantly different dimensions may not accurately approximate an elongated trapped ion cell.

As a side note, the most apparent difference between the profile in Figure $4 a$ and $b$ is a reduction in damping of the observed signal for the external reservoir. Because the primary difference between the two experiments is the orifice size of the interior electrode, a 2-mm diameter conductance limit at $\mathrm{CL}$ in contrast with a $4 \times 12-\mathrm{mm}$ oval shaped orifice at ST, it is concluded that the size of the urifice thruugh which the ions travel is in some way responsible for the damping of the oscillation profile of Figure 4 . One such damping mechanism involves the clipping of the interior electrode by ions formed off axis or by ions formed with a higher velocity component transverse to the $z$ axis. Also, Kerley et al. [26] recently demonstrated ion loss due to axial magnetic inhomogeneities in a two-section FT-ICR cell [26]. It is possible that ions passing through the smaller orifice are in closer proximity to the magnetic inhomogeneities associated with the conductance limit and are thus more likely to be lost by this mechanism than ions passing through the larger oval shaped orifice at ST. It is presumed that a combination of these loss mechanisms contributes to the damping of the oscillation signal in Figure 4. 


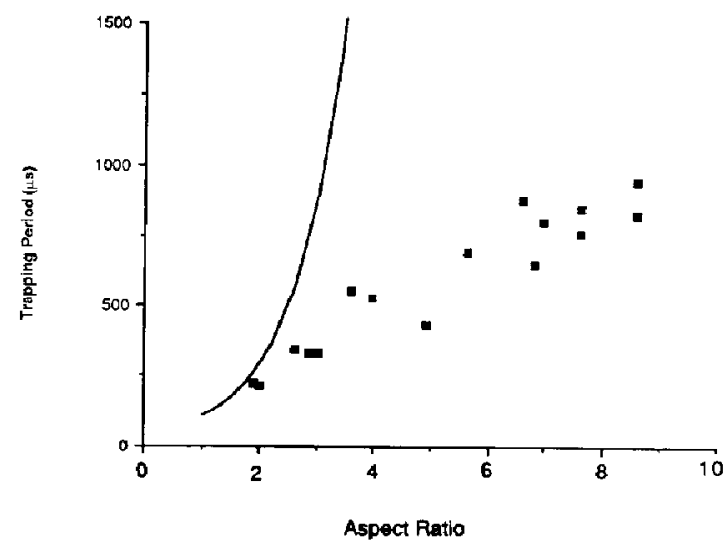

Figure 5. Trapping period data for naphthalene molecular ion in 2.0-V wells, $\square$, of width $5 \mathrm{~cm}$ and length 10 to $43 \mathrm{~cm}$. Also included is the predicted trapping period using equation 9 for $\beta$ as well length increases (solid line).

Oscillations in elongated reservoirs. It was expected that as the cell length grew, increasingly anharmonic motion would be observed as ions of different kinetic energies established motion with increased particle-in-abox character. This anharmonicity would result in a dephasing of the ion signal and preclude measurement of a trapping period by the method employed here. It was surprising then that an oscillating signal continued to be measured as the well length was increased to a limit of $43 \mathrm{~cm}$. For example, as shown in Figure $4 \mathrm{c}$, a trapping period of $870 \mu$ s is clearly evident and, although not shown, extends for several milliseconds with no evidence of broadening or dephasing. The two most likely explanations for this result are either that the wells retain a strongly harmonic character or that ions of a particular kinetic energy are preferentially detected when Giancaspro and co-workers' $[15,16]$ method is used to directly observe trapping frequency. The ion trajectory data in Figure 2 reject the former explanation, leaving the alternative that ions of a particular energy are preferentially detected by FTMS using the method of Giancaspro and co-workers.

As indicated earlier, the quadrupolar approximation for trapping motion is often used in deriving equations of motion in the trapped ion cell because analytical solutions are possible. Equation 9, which is based on this approximation, was previously derived to model trapping oscillations in elongated wells [8]. In that work, $\beta$, the cell geometry factor predicted a rapid increase in trapping periods with increased cell length. This is shown in Figure 5 for the $m / z 128$ ion in a 2.0-V well. However, also included in Figure 5 is a sampling of experimental trapping period data acquired with the suspended trapping pulse sequence shown in Figure 3 . As the length of the well increases from 10 to 43 $\mathrm{cm}$, the trapping period increases, but in a near linear fashion. Equation 9 clearly fails for this type of trapping measurement. We assume that because the quadrupolar approximation is effective near the center of the well, the fact that the data do not adopt a response following the predicted $\beta$ indicates that the detected ion bundle extends closer to the boundaries of the cell, i.e., ions are formed higher in the potential well and possess relatively large kinetic energy at the bottom of the well. A rough estimate of kinetic energy based upon flight time between trap plates is $0.6 \mathrm{eV}$.

As a first measure of the relative merit of the various models for trapping motion, potential maps are compared in Figure 6 for the cosh approximation, the exact solution in eq 1, and expanded Taylor approximations of the potential in eq 6. Potential wells are created for the $5-\mathrm{cm}$ cubic cell and for two elongated cells of dimensions $5 \times 5 \times 13 \mathrm{~cm}^{3}$ and $5 \times 5 \times 38 \mathrm{~cm}^{3}$. For each case $V_{T}=2.0 \mathrm{~V}$ and $V_{0}=0.0 \mathrm{~V}$. As seen in Figure $6 \mathrm{a}$, even for the cubic cell a fourth order Taylor expansion is required to approximate the exact solution. Both the quadrupolar and cosh solutions are in poor agreement, with the quadrupolar solution overestimating the potential at the center of the well by $18 \%$ and the cosh expression underestimating the potential by $36 \%$. This result for the cosh function is not unexpected because it is for the elongated wells that the approximation should excel. This is shown in both Figure $6 \mathrm{~b}$ and $\mathrm{c}$ as the potential well exhibits a steep reduction in potential at the boundaries and a uniform near-zero field region in the center of the well. In contrast, the quadrupolar approximation of the potential is increasingly ineffective and added Taylor terms are required to approach the results of the cosh function model.

Calculations of absolute potentials can be misleading because it is actually the potential gradient that influences ion motions. A more effective demonstration of the cosh function approximation in elongated wells is shown in Figure 7a where for $V_{T}=2.0 \mathrm{~V}$, the trapping periods for a family of curves of increasing ion energy are calculated for wells varying in length from $5 \mathrm{~cm}$ to $38 \mathrm{~cm}$. In contrast with the quadrupolar approximation, which exhibits an invariance of trapping period to ion kinetic energy, at large increases in well sizes the cosh function correctly predicts the reduced period of lower energy ions. As desired, excellent agreement is found between the cosh approximation in Figure $7 \mathrm{a}$ and curves shown in Figure $7 \mathrm{~b}$ that are generated by the ion trajectory model; the most noticeable deviation occurs for lower energy ions in wells with aspect ratios of 2 to 4 .

As presented in the form shown in Figure 7 , the model explains the variation of the experimental data in Figure 5 from the prediction based on eq 9. For ions with large initial total energy, a proportional relationship between period and cell length is indicated. The prediction based upon eq 9 that trapping period increases rapidly with cell length is also indicated by the cosh model, but is limited to ions with small $z$-axis energy. A new family of curves in Figure 8, created from a transposition of data in Figure 7, better illustrates the 

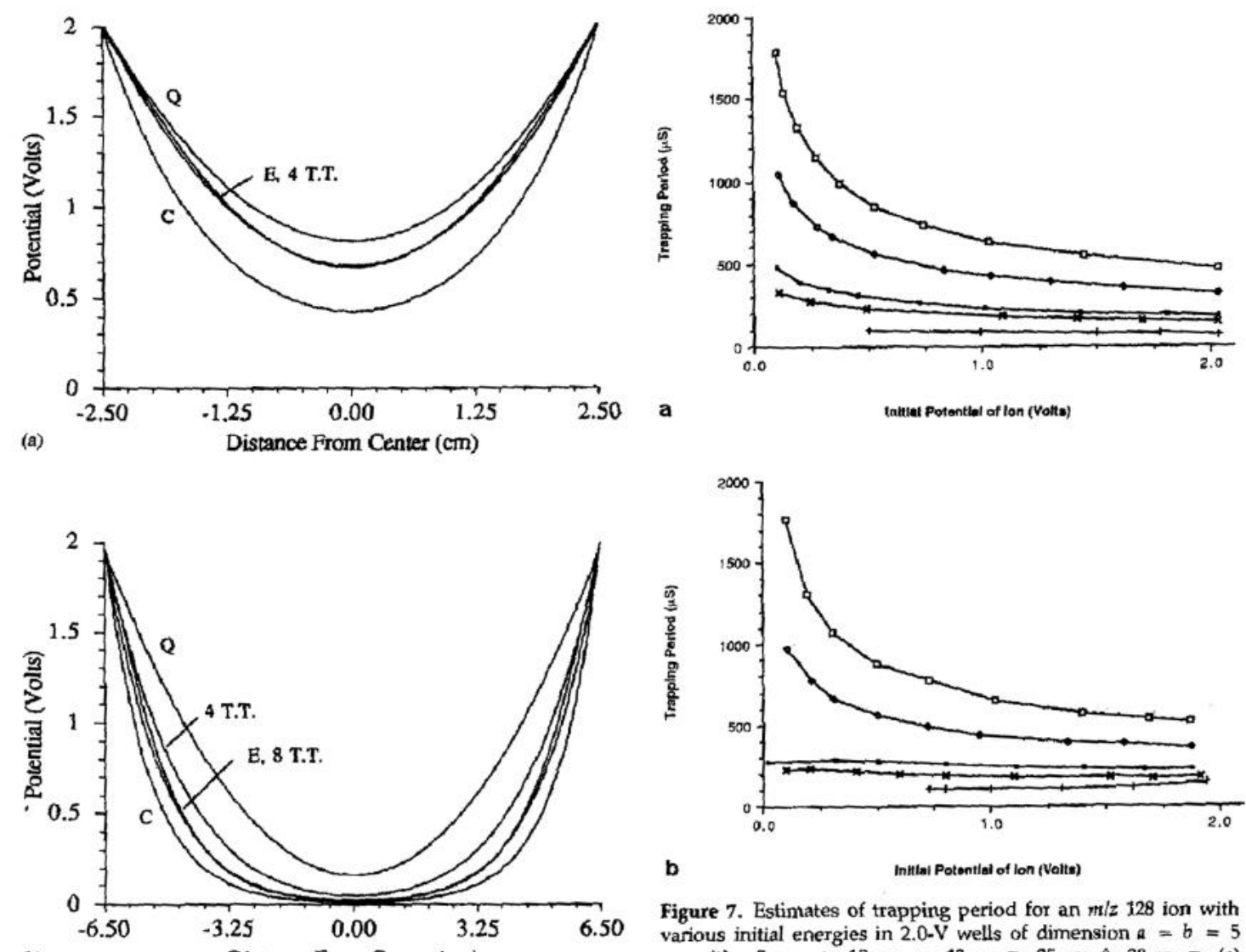

(b) Distance From Center (cm)

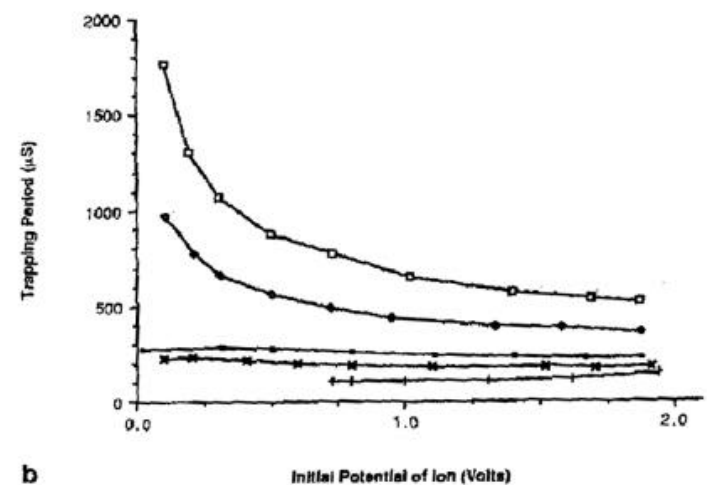

Figure 7. Estimates of trapping period for an $m / z 128$ ion with various initial energies in $2.0-\mathrm{V}$ wells of dimension $a=b=5$ cm with $c: 5 \mathrm{~cm},+; 10 \mathrm{~cm}, x ; 13 \mathrm{~cm}$ - $25 \mathrm{~cm} \diamond ; 38 \mathrm{~cm} \square$. (a) Ion trajectories obtained from the cosh function approximation in eq 18; (b) results from ion trajectory simulations [24].

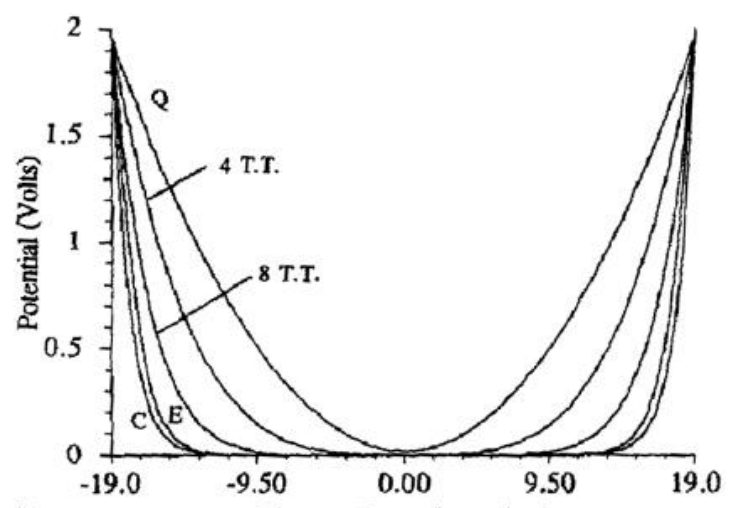

(c)

\section{Distance From Center (cm)}

Figure 6. Potential wells are calculated from the exact solution, $E$, of the potential in eq 1 , expansion of eq 1 as a Taylor series, $Q$ (quadrupolar), 4 T.T. (fourth order), 8 T.T. (eighth order), and the cosh approximation, $C$, for cells of length (a) $5 \mathrm{~cm}$; (b) $13 \mathrm{~cm}$; and (c) $38 \mathrm{~cm}$.

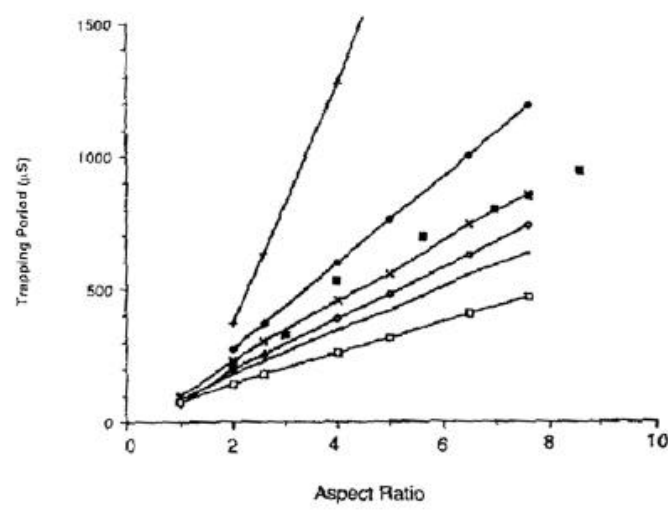

Figure 8. Transposition of data in Figure 7 to develop a family of curves for ions with various initial kinetic energies. Also included are the data from Figure 5 that demonstrate an approximate ion energy of $0.6 \mathrm{eV}$ with the cosh model for trapping motion. 


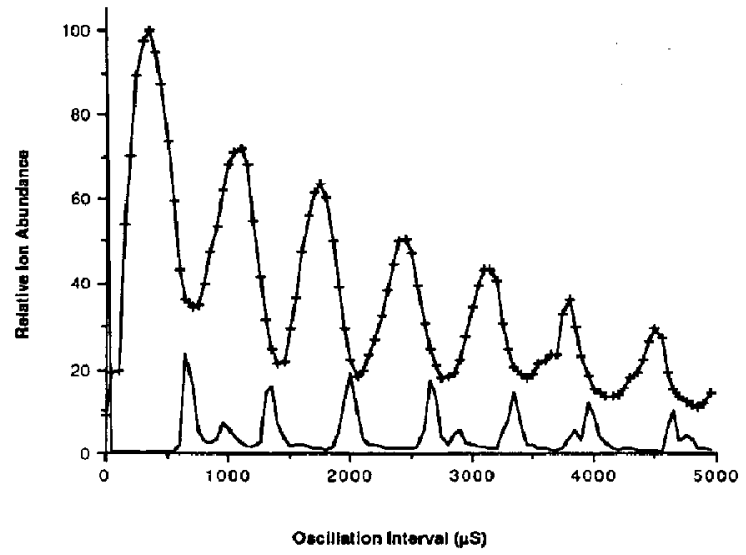

Figure 9. Oscillation profile of $m / z 128$ ions in a $38-\mathrm{cm}, 2.0-\mathrm{V}$ well with ions originating in the external reservoir, + , and originating in the source trap, - In both cases the oscillation occurs between electrodes $\mathrm{CP}$ and $\mathrm{CL}$ with detection in the source trap.

relationship between trapping period and cell length for ions with a specific kinetic energy. When the experimental data from Figure 5 is plotted in this form, ion energies of $0.6 \mathrm{eV}$ are estimated by the cosh function model.

An obvious question to be asked concerns the energy characteristics of ions that are observed. Because monoenergetic ions cannot result from the ionization process, it appears that this pulse sequence produces an ion population distributed about a particular energy that is either more efficiently trapped or detected. Several experiments were attempted in an effort to understand the energetics of such ions. The trapping periods for several ions of PFTBA were shown to vary as expected with the square root of mass. Trapping period was also observed to vary with perimeter trap potentials. Surprisingly, however, no other variation in the experiment design altered the oscillation period. For example, larger ion densities increased signal damping and distorted the oscillation profile, but did not alter the trapping period. In another experiment, at constant perimeter electrode potentials, variation of the potential iritially applied to the interior electrode did not alter the period. Attempts to demonstrate any dephasing of the ion profile were also unsuccessful. For example, profiles were obtained for ions initially formed in the source trap while the reservoir was quenched. Because the average electric field variation experienced by ions is much greater in smaller wells, it was thought that the larger distribution of total ion energies, and thus broader kinetic energy distribution of the ion bundle, would result in a dephasing of the signal or perhaps a change in kinetic energy of the ions detected. However, as the comparison profiles in Figure 9 indicate, this is not the case. The peak width is much narrower, as would be expected for an inn packet formed in a smaller well; but again, the trapping periods are identical. We speculate then that the source of these preferentially detected ions lies not with ion formation or ion equilibration, but rather with the method used for detection.

The two-step suspended trapping sequence in Figure $3 b$ twice alters the kinetic energy distribution of ions, each time leading to selective ejection due to a nonadiabatic change in trap potentials. This $z$-axis ejection mechanism is currently under investigation and is the subject of an upcoming publication [27]. In brief, if an ion in a trapped ion cell has a total translational energy, the sum of its kinetic energy and potential en-

Table 1. Trapping periods in elongated cells with $2.0-\mathrm{V}$ trap potentials

\begin{tabular}{|c|c|c|c|c|}
\hline Kinetic energy $(\mathrm{eV})^{a}$ & Cell length (cm) & $\begin{array}{l}\text { Trappin } \\
\text { Exact }^{\text {b }}\end{array}$ & $\begin{array}{l}\text { Ood }(\mu s) \\
\operatorname{Cosh}^{c}\end{array}$ & Quadrupolar ${ }^{d}$ \\
\hline 0.03 & $\begin{array}{r}5 \\
10 \\
25 \\
38\end{array}$ & $\begin{array}{r}110 \\
270 \\
1700 \\
2480\end{array}$ & $\begin{array}{r}122 \\
347 \\
1759 \\
2947\end{array}$ & $\begin{array}{c}109 \\
297 \\
8,260 \\
1.48 \times 10^{5}\end{array}$ \\
\hline 0.1 & $\begin{array}{r}5 \\
10 \\
25 \\
38\end{array}$ & $\begin{array}{r}110 \\
250 \\
975 \\
1770\end{array}$ & $\begin{array}{r}120 \\
307 \\
1042 \\
1784\end{array}$ & $\begin{array}{c}109 \\
297 \\
8,260 \\
1.48 \times 10^{5}\end{array}$ \\
\hline 0.75 & $\begin{array}{r}5 \\
10 \\
25 \\
38\end{array}$ & $\begin{array}{l}110 \\
195 \\
480 \\
775\end{array}$ & $\begin{array}{l}105 \\
200 \\
484 \\
739\end{array}$ & $\begin{array}{c}109 \\
297 \\
8,260 \\
1.48 \times 10^{5}\end{array}$ \\
\hline 1.50 & $\begin{array}{r}5 \\
10 \\
25 \\
38\end{array}$ & $\begin{array}{l}- \\
175 \\
380 \\
565\end{array}$ & $\begin{array}{r}94 \\
163 \\
386 \\
545\end{array}$ & $\begin{array}{c}297 \\
8,260 \\
1.48 \times 10^{5}\end{array}$ \\
\hline
\end{tabular}

- Kinetic energy of ion at center of well.

- Data extracted from SIMION trajectory program [24].

- Data acquired using eq 18.

Data acquired using eq 21 from ref 8. 
ergy, which exceeds the maximum depth of the potential well, it will escape the cell. This phenomenon occurs when an ion enters a trapped ion cell in which trapping potentials are to be raised nonadiabatically as in gated trapping experiments. If the ion is close enough to the trap plate when the potential is raised nonadiabatically, it may experience a sufficient gain in potential energy to cause ejection by this mechanism or an increase in $z$-axis trapping amplitude. Moreover, during excitation $z$-axis ejection is prevalent for particular populations of ions. We are presently modeling the suspended trapping experiment to ascertain if a single energy ion is preferentially retained or detected by FTMS for the pulse sequence in Figure 3.

\section{Conclusion}

Regardless of the source of the ions, the cosh model in eq 18 is a clearly superior choice for estimating trapping periods in elongated wells, as the tabulation of trapping periods in Table 1 demonstrates. The quadrupolar model does not account for a variation in initial ion kinetic energy and, moreover, grossly overestimates trapping period for large wells. Only for the cubic cell, which exhibits a strong harmonic character, is the model adequate. In contrast the cosh model correctly tracks the exact solution for cubic and elongated cells for ions with any initial axial energy. Wurst case results are for low energy ions in wells of moderate length with errors approaching $30 \%$. Average errors for 5-, 10-, 25-, and 38-cm wells at the four tabulated energies were $8.2 \%, 14.6 \%, 3.1 \%$, and $6.6 \%$, respectively. Equation 18 is an effective alternative to the harmonic oscillation expression for approximating $z$-axis trapping motion in rectangular trapped ion cells. Especially for elongated cells or for ions with nonthermal kinetic energies, the cosh approximation or a numerical solution of eq 11 must replace solutions based on the quadrupolar approximation to avoid large errors in the modeling of trapping motion.

\section{Acknowledgments}

The authors would like to thank the reviewers for many helpful comments, and especially Peter Grosshans for his suggestion of eq 15 as an alternative to the potential gradient of eq 14. This work is supported by the Welch Foundation and by a grant from the Texas Advanced Technology and Research Program.

\section{References}

1. Ledford, E. B. Ir.; McIver, R. T., Ir. Int. I. Mass Spec. Ion Physics, 1976, 22, 399-406.

2. Ledford, E. B. Jr.; Ghaderi, S.; White, R. L.; Spencer, R. B.; Kulkarni, P. S.; Wilkins, C. L.; Gross, M. L. Anal. Chem. $1980,52,463-468$.

3. Ledford, E. B. Jr.; Rempel, D. L.; Gross, M. L. Anal. Chem. 1984, 2744-2748.

4. Grosshaus, P. B.; Wang, M.; Marshall, A. G. 36th Annual Conference on Mass Spectrometry and Allied Topics, 1988; pp 592-593.

5. Wang, M.; Marshall, A. G. Anal. Chem. 1989, 61, 1288-1293.

6. Rempel, D. L.; Ledford, E. G. Jr.; Huang, S. K.; Gross, M. L. Anal. Chem. 1987, 59, 2527-2532.

7. Wang, M.; Ledford, E. B. Jr.; Marshall, A. G.; FACSS XIV, Detroit, MI, October, 1987, abstract $\# 43$.

8. Hunter, R. L.; Sherman, M. G.; McIver, R. T. Jr.; Int. J. Mass Spectrom Ion Proc. 1983, 50, 259-274.

9. Rempel, D. L.; Huang, S. K.; Gross, M. L. Int. J. Mass Spectrom. Ion Proc. 1986, 70, 163-184.

10. Hanson, C. D.; Kerley, E. L.; Castro, M. E.; Russell, D. H.; 37th Annual Conference on Mass Spectrometry and Allied Topics, 1989; pp 1274-1275.

11. Wang, M.; Marshall, A. G.; 37th Anmual Conference on Mass Spectrometry and Allied Topics, 1989; PP 1226-1227.

12. Van De Guchte, W. J.; Van Der Hart, W. J. Int. J. Mass Spectrom. Ion Physics 1990, 95, 317-326.

13. Beauchamp, J. L.; Armstrong, J. T. Rev. Sci. Instrum. 1969, $40,123$.

14. Schweikhard, L.; Blundschling, M.; Jertz, R.; Kluge, H.-J. Int. J. Mass Spectrom. Ion Proc, 1989, 87, R7-R12.

15. Giancaspro, C.; Verdun, F. R. Anal. Chem. 1986, 58, 2097-2099.

16. Giancaspro, C.; Verdun, F. R.; Muller, J.-F. Int. J. Mass Spectrom. Ion Proc. 1986, 72, 63-71.

17. Hanovich, J. P.; Markey, S. P. 36th Annual Conference on Mass Spectrometry and Allied Topics, 1988; pp 606-607.

18. Kofel, P.; Allemann, H.-P.; Wanczek, K. P. Int. J. Mass Spectrom. Inn Proc. 1985, 6.5, 97-103.

19. McIver, R. T. Jr.; Hunter, R. L.; Bowers, W. D. Int. J. Mass Spectrom. Ion Proc. 1985, 65, 67-77.

20. Laude, D. A. Jr.; Beu, S. C. Anal. Chem, 1989, 61, $2422-2427$.

21. Hofstadler, S. A.; Laude, D. A. Jr. Int. J. Mass Spectrom. Ion Proc. 1990, 97, 151-164.

22. Hogan, J. D.; Laude, D. A. Jr. Anal. Chem., in press.

23. Sharp, T. E.; Eyler, J. R.; Li, E. Int. J. Mass Spettrom. Ion Physics 1972, 9, 421-439.

24. Dahl, D. A; Delmore, J. E. SIMION PC/PS2 version 4.0; Idaho National Engineering Laboratory (EGG-CS-7233, Rev. 2), April 1988.

25. Chesnavich, W. J.; Su, T.; Bowers, M. T. J. Chem. Physics $1976,65,990-997$.

26. Kerley, E. K.; Hanson, C. D.; Castro, M. E.; Russell, D. H. Anal. Chem 1989 61, 2528-2534.

27. Hofstadler, S. A.; Laude, D. A. Jr. Int. J. Mass Spectrom. Ion Proc. accepted for publication, 